\title{
Splint Designed to be used in the "Pre- and Post-Operative" Correction of Pectus Excavatum with the Nuss Procedure, Pectus Press (PP)
}

\author{
Campos JRM*, Miranda Luzo MCD, Iamamura AY, Tedde ML and \\ Fernandes PP \\ Department of Thoracic Surgery and Occupational Therapeutic, University of Sao \\ Paulo, Brazil
}

\section{Mini Review}

Volume 2 Issue 3

Received Date: July 10, 2018

Published Date: July 24, 2018

*Corresponding author: Jose Ribas Milanez de Campos, Department of Thoracic Surgery and Occupational Therapeutic, University of Sao Paulo, Brazil, Tel: 5511983351889; Email: jribas@usp.br

\section{Abstract}

Objective: Evaluation of effectiveness of the use of the Pectus Press (PP) on patients with Pectus Excavatum (PE) as initial and post-operative treatment of the Nuss procedure for the correction of posture, reduction of pain and prevention of bar displacement.

Methods: Between August 2015 and December 2017, 159 patients, from 7 to 46 years (M=17), with PE were treated. On first visit patients received the orthotic equipment and oriented to follow a home exercise program and physical activities. Thirty-six (64.4\%) underwent surgery during this period of time. We checked the correct size, position of the supports, the pressure of the shoulder straps on each visit every 30-60 days. The Valsalva maneuver with a "Pectus Whistle" after October-2016 was also recommended.

Results: Better posture and more flexibility of the thorax were observed by patients and parents after the first three weeks including reduction of costal flaring in 73 (84\%) that used the device for more than 12 hours a day including nighttime use. In the first 30 - 90 days of post-operative period, helped to reduce the pain and the possibility of bar bending, with the maintenance of a corrected posture. No bar displacement was observed in the operated group and less pain medication were prescribed.

Conclusions: Treatment using orthosis and exercises for PE has been very well described (Haje Method)."Pectus Press" is a flexible low-profile orthosis and has shown to be a useful tool in the pre- and post-operative periods of the Nuss procedure. Adding exercise's + Pectus whistle's, it provides thoracic chest wall flexibility. In the post-operative period, less pain and no bar displacement were observed. The success of treatment was directly linked to the patient age, time and frequency of the use of the device. 
Keywords: Pectus Press; Pectus Excavatum

Abbreviations: PP: Pectus press; PEX: Pectus excavatum

\section{Introduction}

Pectus excavatum (PEX) is a deformity usually detected in early childhood and becomes more evident with the growth. There is no index, or any indication of prognosis as a basis for progression or degree of deformity development. Clinical and armed workup assessments can demonstrate significant changes in cardiac and lung physiological index in the deep, asymmetric and important deformities. However, most of these patients have severe psychological disorders due to the irregular contour of the chest. They tend to withdraw, not expose themselves in public, avoid sports activities, relationships; interact among colleagues and all or any situations that require exposure of his deformity. Psychological changes have been identified including the parents, who begin to consider their children unable to carry out normal physical activities. All these changes associated, end up contributing and causing deep and progressive postural deformities, often associated with scoliosis, postural defects, ante-version shoulder [1].

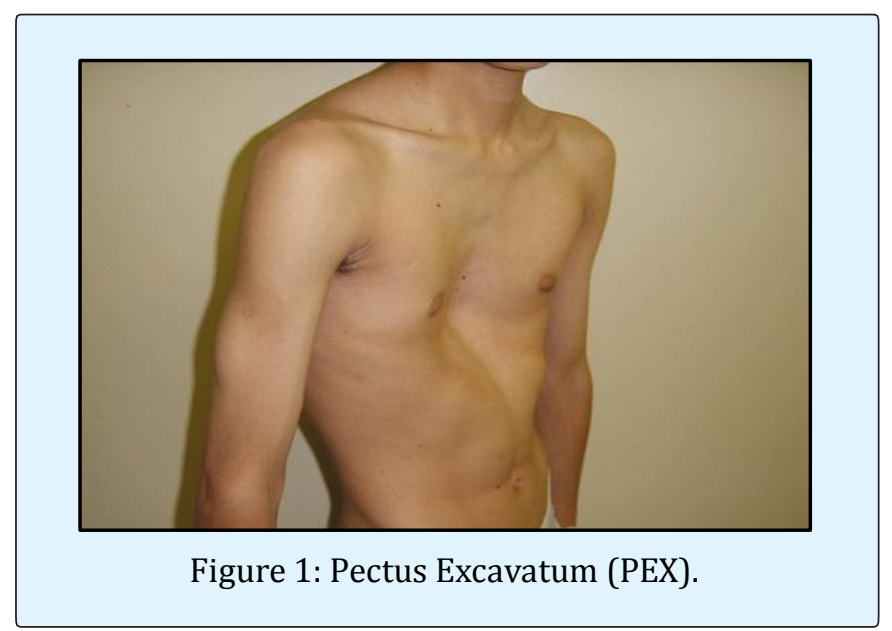

Currently reported results and descriptions of clinical or conservative treatment suggesting skeletal remodeling through the use of orthosis compressing the highest portions of the meshed previous costal ribs using these technics have been described several years ago and some surgeons and services consider questionable, especially when it comes to the retraction of deformities, such as PEX and recently there is an increase of favorable results published. Most patients are asymptomatic, and the main goal of treatment with orthosis, in general, is to improve clinical, postural and psychological condition through the possibility to correct the deformity of the chest wall. In many cases, there is even a considerable improvement of the patient's ability to exercise.

The ideal time to indicate the surgical correction is discussed, although most authors agree that it should not be done in patients with less than five years, as this could cause abnormalities in the growth of the thoracic framework. Currently, it is almost a consensus disseminated by different services that make this type of surgery, that it's wiser to indicate the surgical treatment during puberty, for orders than 10 or 12 years, when most of physical and psychological changes occurs [2].

Regarding the best time to indicate and start treatment with the use of orthosis, we believe that undouble it is as soon as possible, and this is one of the biggest advantages of Pectus Press (PP), it can be used immediately after the first evaluation. The device is available to almost all ages and sizes. The patients and for the parents who start the use of the device notice the benefits of postural correction, improve of flexibility of the rib cage and the beginning of physical activity practice also contribute to increase patient adherence that leads to best results.

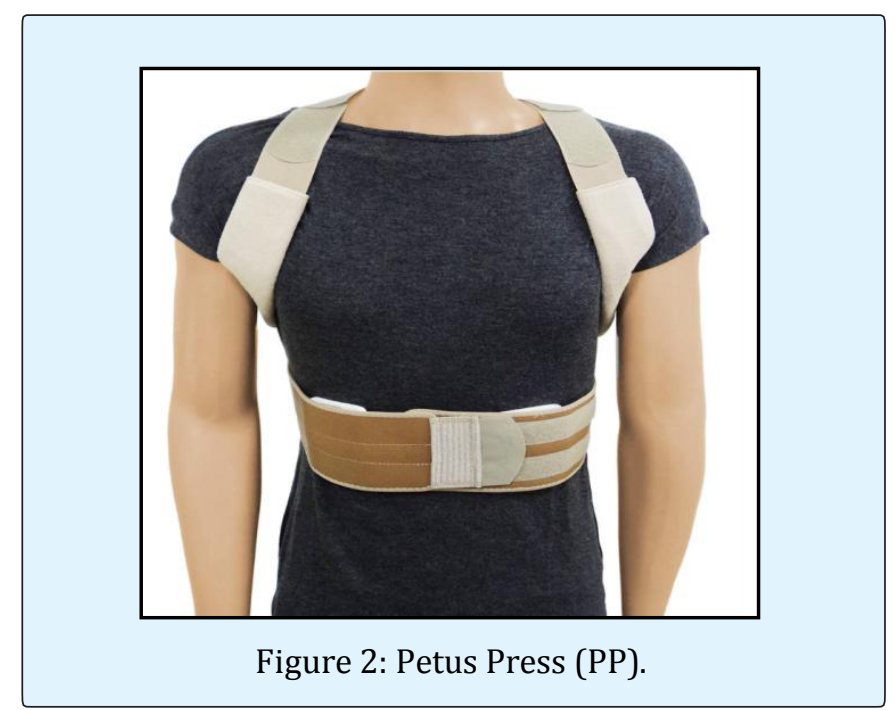




\section{International Journal of Surgery \& Surgical Techniques}

\section{Objective}

The objective of this paper is to evaluate the effectiveness of the use of the Pectus Press (PP) in patients with Pectus Excavatum (PEX) as initial and postoperative of the Nuss procedure on correction of posture, reduction of pain and bar displacement prevention. A medical or occupational therapy supervision is usually required (Figure 2).

The indications for the orthosis for the initial treatment for patients with PEX who will be operated with the Nuss procedure, has the mainly characteristics: correction and preservation of the posture in the thoracic hypnosis and as in the majority of the patients with PEX associated with shoulder ante-version. The prominent costal flaring could also be treated, preferable if stated as soon as possible at the first visit. Another indication is for the post-operative period during the first 30 - 90 days, it helps to reduce pain and prevention of bar bending, with the maintenance of a better and correct posture.

The orthosis is a single piece and of easy fitting, it is composed of axillary padding, a back rest in rigid plastic for thoracic support, comfortably covered by cushioned textile. Precautions must be taken to check the correct size (Figure 3), the position of the thoracic supports, because the excessive pressure of the shoulder straps that may induce discomfort, pain and rarely circulatory problems.

\begin{tabular}{|c|c|c|c|}
\hline \multicolumn{4}{|c|}{ SIZE-cm } \\
\hline & \multirow[t]{2}{*}{ Back height-cm } & \multicolumn{2}{|c|}{ Circumference - cm } \\
\hline & & Minimum & Maximum \\
\hline Extra small & 28 & 56 & 64 \\
\hline Small & 33,5 & 62 & 74 \\
\hline Medium & 35 & 70 & 84 \\
\hline Large & 36 & 80 & 94 \\
\hline Extra large & 37 & 94 & 111 \\
\hline Extra extra large & 38 & 105 & 130 \\
\hline \multirow[t]{2}{*}{ Measures } & back height & \multicolumn{2}{|c|}{$15 \mathrm{~cm}$ above the waist } \\
\hline & circumference & \multicolumn{2}{|c|}{$15 \mathrm{~cm}$ above the waist } \\
\hline
\end{tabular}

Instructions for Use: (Figure 4)

1 - Release closure straps of thoracic waist;

2 - Place arms through shoulder straps;

3 - Adjust straps paddings at axillary level;
4 - Place thoracic supports over the prominent ribs and fasten adherent straps to close thoracic belt;

5 - Adjust shoulder straps;

6 - Can be used under or over a comfortable cotton TShirt.

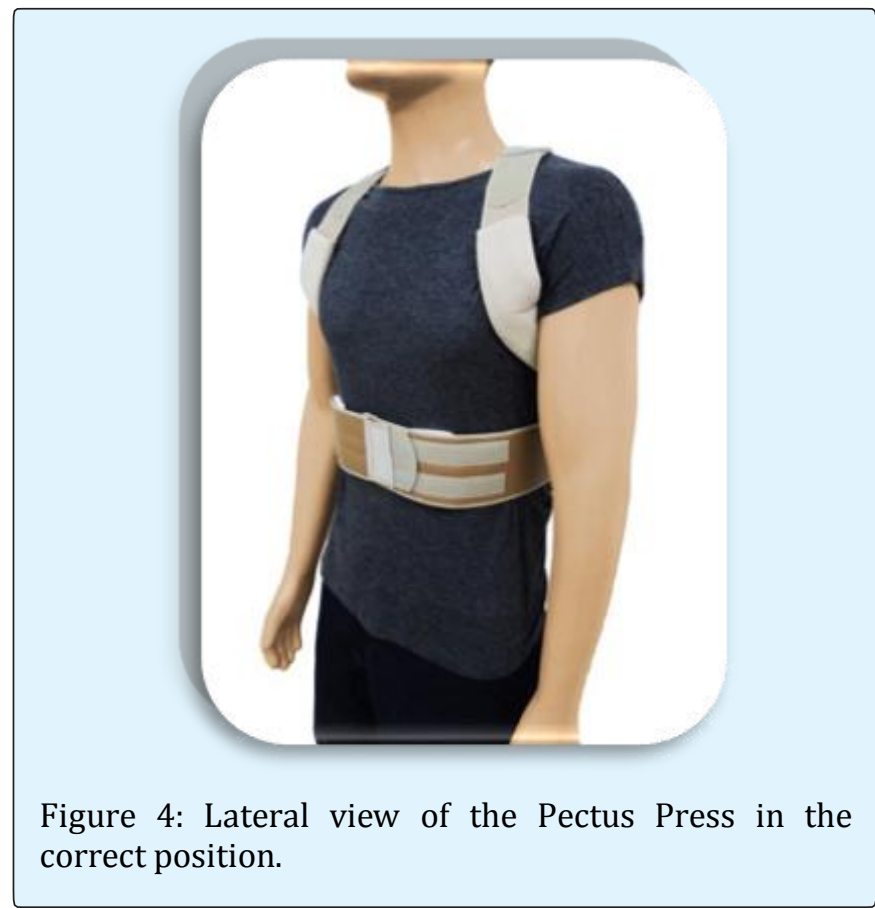

\section{Historic Review and Actual Indications}

Based in the lessons learned from Prof. Sidney Haje [3], his great legacy in Medicine was the creation of a conservative treatment protocol for chest-wall deformities. He treated his first patient more than 35 years ago, when he created the technique of Dynamic Chest Compressor Brace, in association with a protocol of specific exercises (Dynamic Remodeling Method), which set new standards for the treatment of Pectus carinatum and Pectus excavatum. He also pointed out that for each type of pectus; there is a treatment, a prognosis, and an ideal age for beginning and follow-up the treatment. The thoracic conformation and flexibility of the chest wall is variable and important for all techniques in the clinical and surgical management. More recently, after 2006, the real statement from the Haje Method was based on the simultaneous practice exercises that promote increased intrathoracic pressure. This method involves balance of forces on the chest, with the respiratory movements in and out, while orthotics exerts a dynamic external pressure on protruding or bulging areas, so providing the remodeling of the ribcage as a whole. The method always 


\section{International Journal of Surgery \& Surgical Techniques}

requires proper and prolonged medical supervision because it can take a year or more for a correction to stabilize.

Among the difficulties we found on the use of the HAJE method of treatment, was the individual orthotics fabrication. Another difficulty is on mounting the aluminum bars, to allow adjustments to the progression of pressure, patient's growth and chest changes by treatment. The screws that serve as compression mechanism by lateral thread in these rods, that provide the dynamic force, represent a barrier on the use, as they promote discomfort and are responsible for a large number of resistances and or abandonment of treatment due to system rigidity.

The orthosis we propose is flexible, has some elasticity and is low profile. As these characteristics influence in comfort, it can be used in contact with the skin or over a thin clothes. The adjustment is easy and not complex to the patient to wear. For example, the continuous pressure of the device over the flare ribs by the front pads, promote remodeling of soft tissue and changes the breathing pattern of the patient, bringing air to upper thorax and uses the lungs as correction motor by stretching the small intercostals muscles that act as deformity forces. With the use of PP, we have noticed a low rate of abandon of the orthosis and a high rate of adherence as patients use, as recommended, including during the sleeping time. It also doesn't influence in activities such as sports, social interaction and keep the correct posture during studying hours, on the use of computers and other leisure and/or other static position activities such as playing video games.

Along with the use of the orthosis, is very important a sequence of exercises complements the dynamics of treatment. An instructional booklet is provided and explains to the patient and their caregivers, an exercise series that include stretching, strengthening and the Valsava maneuver with number of repletion's in a daily program. Added to that, exercising with the orthotics is essential. Exercises in gyms, team sports, and gymnastics in schools, soccer, volley, basketball and or swimming should also be encouraged and performed at least twice a week. The prescription should emphasize that such exercises have as their primary goal not marked muscle hypertrophy, but the movement of the rib cage, the movement of the muscles of the thoracic chest wall. The orthosis in the correct position also helps with the correct breathing.
We emphasize the long-term treatment, where gradual refurbishment of rib cage can be noticed. The patient is instructed to wear the orthotics progressively increasing use in an hour a day, up to 6-7 hours, after this the night time use can be started. We recommend daily use and the more time possible, as patient begins to notice the improvement of the appearance of the thoracic contour, this serves as stimulus and longer use.

The patient should also be instructed to loosen or even to remove the orthosis, in case of discomfort or sharp pain, skin changes, bruises, blisters and or irritated surfaces, and immediately report to the doctor these facts and after relief of symptoms, they must return for the treatment. Progress on the compression rate should happen according to patient tolerance. We have rarely indicated medication for pain control and use of topical medications such as creams or ointments in case of irritations.

Monitoring should be done every two or three months in the first year and a little more spaced every four months in the following years. Eventually, the switch to a new orthosis may be necessary due to the rapid and accelerated growth of some teenagers in the puberty. In the specific case of PP can also make necessary change in position, size and shape of pads used in costal margin, evaluable for easy adaptation to the best compression site.

Between August 2015 and December 2017, a total of 159 patients, from 7 to 46 years $(M=17)$, (children and adults) with PEX were treated with PP in the Division of Thoracic Surgery and Occupational Therapy (University of São Paulo, Brazil). On first visit patients received the orthotic equipment and were oriented to follow a home exercise program and physical activities. Thirty-six $(64.4 \%)$ patients underwent surgery with the Nuss procedure during this period of time. The main characteristics of the observed treatment with PP are: correction and preservation of posture avoiding thoracic hypnosis, associated shoulder ante-version and light circular compression and extra pressure over the prominent costal flaring. All patients easily adapted to this single piece with axillary padding and also the backrest rigid plastic. All parts have confortable cushioned supports. We also checked the correct size, position of the thoracic supports and the pressure of the shoulder straps on each visit every 30-90 days [4,5]. 


\section{International Journal of Surgery \& Surgical Techniques}



"Valsalva Maneuver", with a deep and forced expiration with your mouth closed, uses your left or right hand; try to push the anterior part of the chest wall as far as possible. At least 3 times a day, morning, afternoon, at night, 10 exercises in each section, but if you can do more, better for increase the elasticity and help in the final results. Since last month, our patients are using the new release: a Pectus Whistle (PW), after October-2016, where no sound is emitted, but is very helpful and an good incentive to the patients trying simulate a the Valsalva maneuver, maintained for 3 to 4 seconds, a moderately forceful attempt exhalation against a closed this special device that simulated a closed airway, usually done by closing one's mouth (Figure 5).

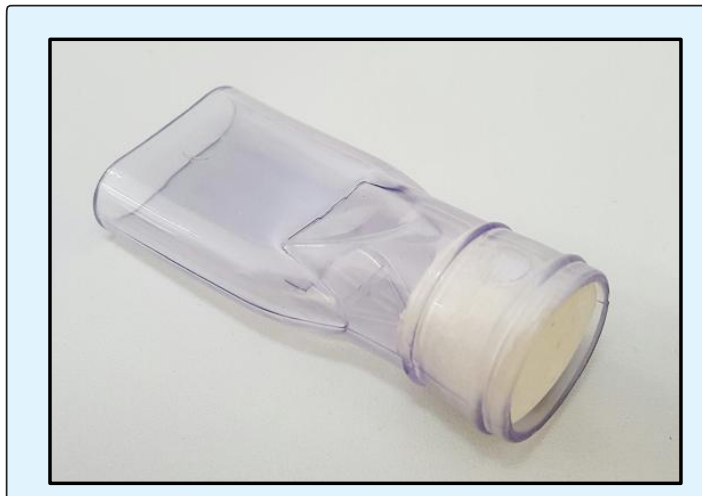

Figure 5: Pectus Whistle (PW).

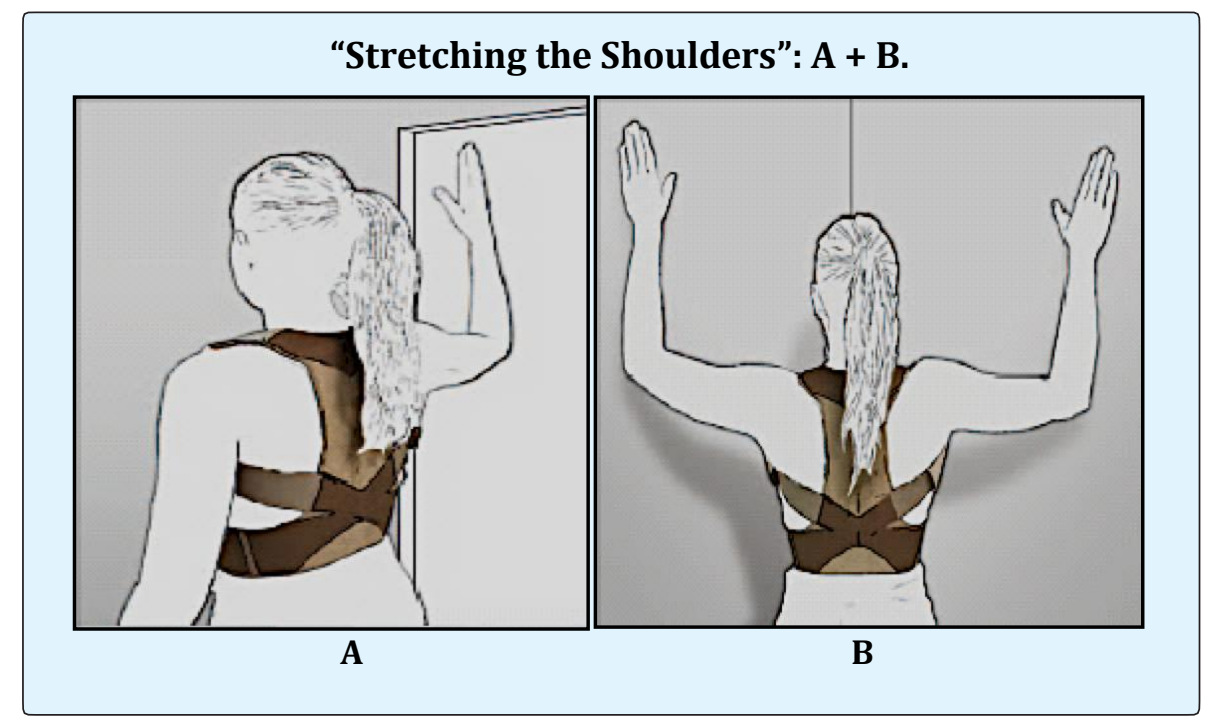


A- Place your hand and elbow on the corner of a wall slowly rotation your body until you feel a slight discomfort. Rotate heads to look over the opposite shoulder Tyr to maintain this stretching position for one minute. Turn and repeat on the other side.
B- Place both hands on the corner of a wall, stabilize your hips and try to place your chin as close as possible to the corner.

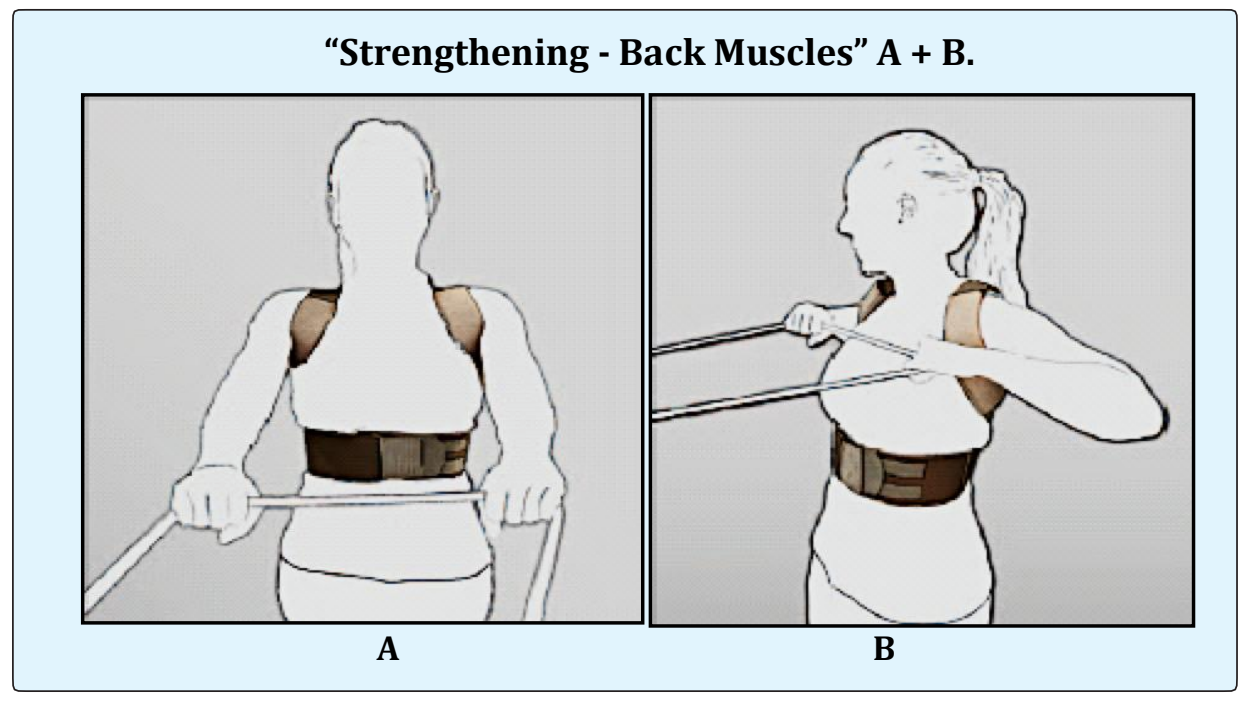

A- With an elastic band attached to the door knob, stand facing the door and hold band with both hands at waist level. Pull it bending your elbows, extending shoulders and feel the scapula pushing together. Try to maintain the

B- Repeat the same exercise with hands. Place your hands higher, at chest level. Maintain the position for 30 seconds or more. position for 30 seconds or more.

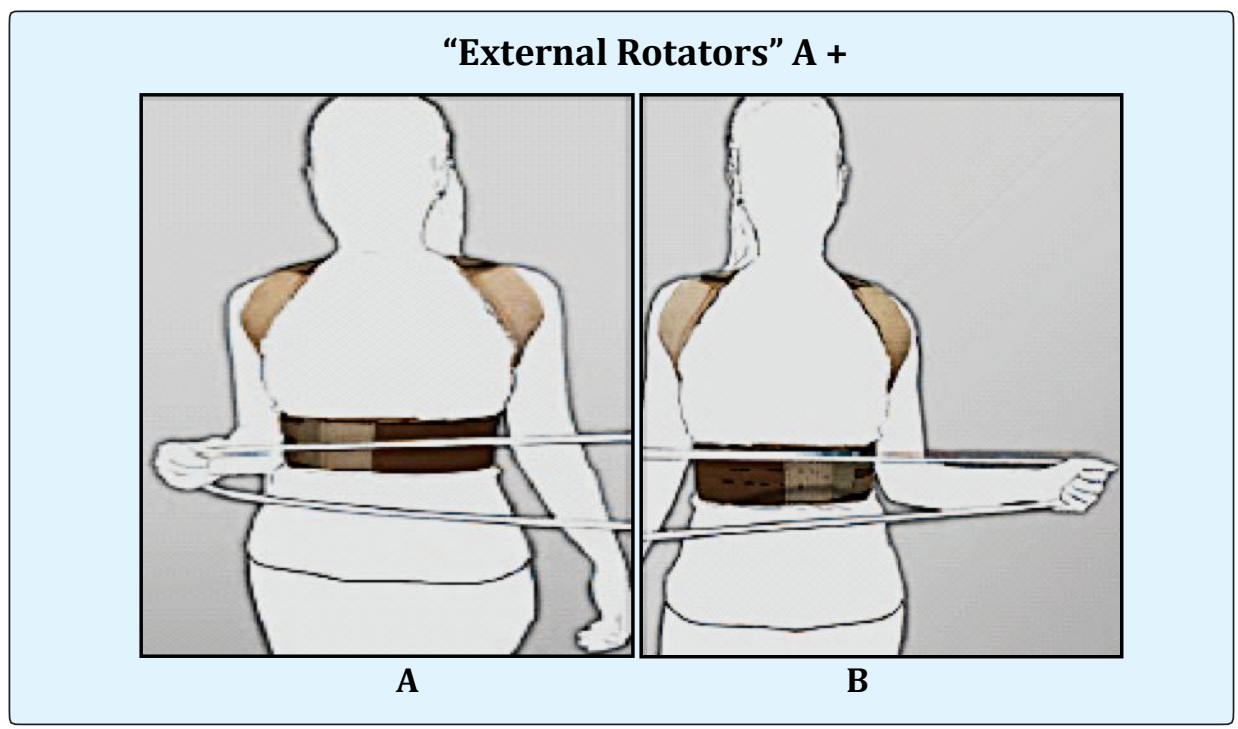

A + B: With an elastic band attached to the door knob, stand beside with elbow flexed at $90^{\circ}$ and arms touching your body. Hold the band and pull it to the side in a swinging motion, while maintaining the arms close to body. Do this with both arms. 


\section{International Journal of Surgery \& Surgical Techniques}

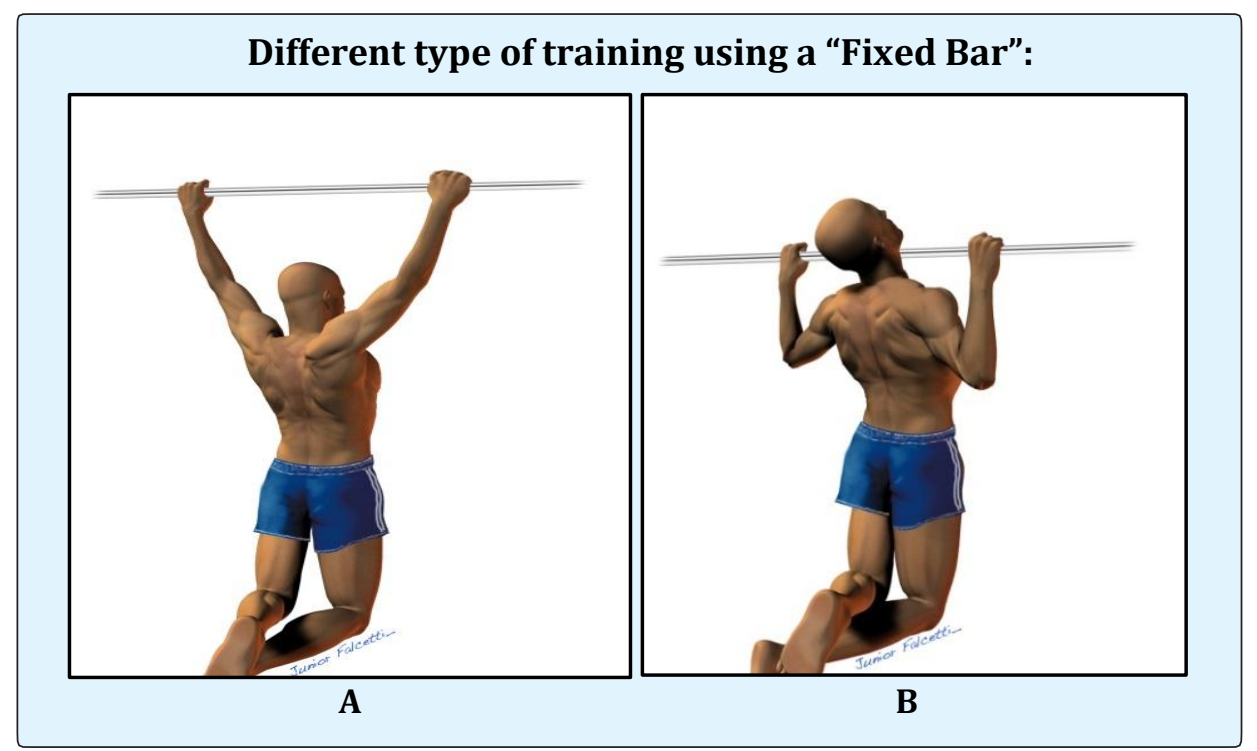

A - First hold with both hands the bar above your head, the distance between a hands and they should be slightly larger than the width of your shoulders, let your weight stretch the lower back muscles for 10 to 20 seconds, rest for a minute and repeat the exercise.

B - With the palms are turned to you, grab the bar and try to move your body upward. These will strengthen the lower back muscles. Breathe when your body is at the bottom; then take a very large inspiration and pull the whole body up. Repeat the exercise comfortably. Plan to do a modest number initially.

Due to the degree of difficulty, the fixed bar is generally avoided by requiring excessive force. On the other hand, it means that there are great physical progress to those who continue to gain muscle mass, strengthening back and arms in a short period of time, which is important for your treatment.

\section{Results of Our Pilot Study}

Better posture and more flexibility of the thorax were observed by patients and parents after the first three weeks including reduction of costal flaring in 73 (84\%) that used the device for more than 12 hours a day including nighttime use. In the first 30-90 days of postoperative period, it also helped to reduce the pain and the possibility of bar bending, with the maintenance of a better corrected posture of patients. No bar displacement was observed in the operated group and less pain medication was prescribed after discharge from hospital during this initial period of follow-up (Figure 6).


Figure 6: Example of one patient using PP in the post-operative period. 


\section{International Journal of Surgery \& Surgical Techniques}

\section{Conclusion of this Pilot Study}

The treatment using the orthotic equipment named Pectus Press, for Pectus excavatum patients in the pre and post-operative period of the Nuss procedure has been successfully done. The PP is a flexible low profile orthosis, a promising alternative and has shown to be a useful and important tool. Special attention for all exercises prescribed with the use of the equipment, must be followed and supervised periodically for specialists, occupational therapists and thoracic surgeons. It provides better posture, increase the thoracic chest wall flexibility; less pain and no bar displacement was noticed in this pilot study, until now, with a short period of time. The success of treatment was directly linked to the patient age, time and frequency of use of the device. The following picture is to exemplify another patient, using PP for six months, more than 10 hours a day including sleeping with it in the night time, to show the results over the costal flaring, according the patient and his parents now $90 \%$ better than was at the beginning of the treatment. Figure $7 \mathrm{~A} \& 7 \mathrm{~B})$.
A

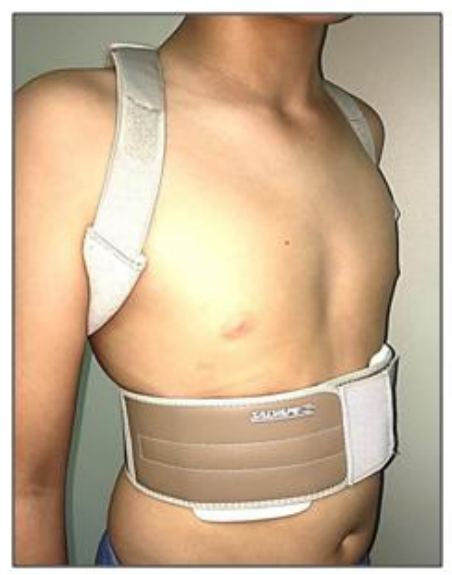

B

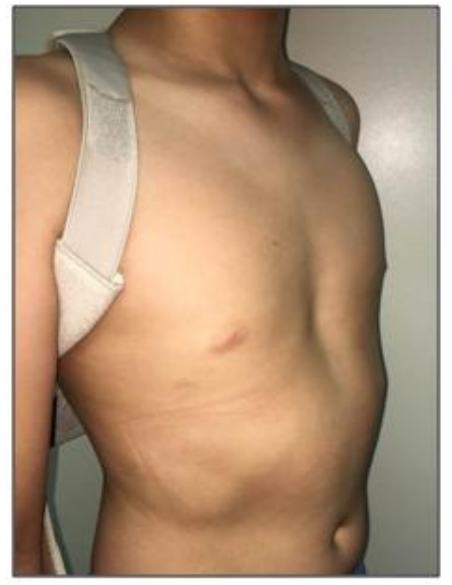

Figure 7: $\mathrm{A}+\mathrm{B}=$ Final aspect of the costal flaring after 6 months of treatment.

\section{Miscelaneous}

Proper treatment in most cases allows the continuity of the treatment and improves the patient's condition. In the case of the patients that were submitted to the Nuss procedure, the main concern was with the displacement of the bars of its proper location in special during the first 30 days. Fortunately this occurrence is not common and maintaining a correct posture, facilitates adequate healing and final fixation of the bar in a permanent position with fibrous tissue throughout the treatment period [6]. In our Service we use the following guidance and care about the sports activities of the post-operative for all patients: a) in the immediate postoperative period on bed rest, drive block, prevent lateral decubitus, trunk flexion and or physical effort with the upper limbs; b) respiratory therapy should be initiated soon after waking up, under the guidance of physiotherapist, maintaining frequency of 2 to 3 times a day until discharge from the hospital; c) after a week, start with active movement of the upper limbs, ambulate often keep trunk rotation in block; d) it is recommended that this time the possibility to use corrective orthotic posture, PP should be used the largest possible number of hours, including and especially during sleeping time. In regular bases this approach is valid for a minimum of 3 months; e) exertion should only be initiated after two weeks postoperatively and almost normal activities after the first month without intense physical exertion; f) totally normal physical activities after 6 months, these activities should be encouraged that even with minimum frequency of $2 \mathrm{x}$ per week and remain so until the end of treatment $[7,8]$.

\section{References}

1. De Ugarte DA, Choi E, Fonkalsrud EW (2002) Repair of recurrent pectus deformities. Am Surg 68(12): 1075-1079. 
2. Fonkalsrud EW, Beanes S, Hebra A, Adamson W, Tagge E (2002) Comparison of minimally invasive and modified Ravitch pectus excavatum repair. J Pediatr Surg 37(3): 413-417.

3. Haje SA, de Podestá Haje D (2015) orthopedic approach to pectus deformities: 32 years of studies. Rev Bras Ortop 44(3): 191-198.

4. Nuss D, Kelly RE Jr, Croitoru DP, Katz ME (1998) A 10-year review of a minimally invasive technique for the correction of pectus excavatum. J Pediatr Surg 33(4): 545-552.

5. Hebra A, Swoveland B, Egbert M, Tagge EP, Georgeson $\mathrm{K}$, et al. (2000) Outcome analysis of minimally invasive repair of pectus excavatum: review of 251 cases. J Pediatr Surg 35(2): 252-257.
6. Nuss D, Croitoru DP, Kelly RE Jr, Goretsky MJ, Nuss KJ, et al. (2002) Review and Discussion of the Complications of Minimally Invasive Pectus Excavatum Repair. Eur J Ped Surg 12(4): 230-234.

7. Campos JRM, Velhote MC, Jatene FB, Fonseca $\mathrm{MH}$, Werebe Ede C (2006) Technical modifications of the Nuss operation for the correction of pectus excavatum. Clinics 61(2): 185-186.

8. Tedde ML, de Campos JR, Wihlm JM, Jatene FB (2012) The Nuss procedure made safer: an effective and simple sternal elevation maneuver. Eur J Cardiothorac Surg 42(5): 890-891. 